\title{
Introduction to the Minitrack Digital Nudging: Online Decision Making and Information Systems Design
}

\author{
Markus Weinmann \\ Department of Information \\ Systems \\ University of Liechtenstein \\ markus.weinmann@uni.li
}

\author{
Christoph Schneider \\ Department of Information Systems \\ College of Business \\ City University of Hong Kong \\ christoph.schneider@cityu.edu.hk
}

\author{
Jan vom Brocke \\ Department of Information \\ Systems \\ University of Liechtenstein \\ jan.vom.brocke@uni.li
}

\section{Introduction}

Research in behavioral economics, as well as psychology, has repeatedly demonstrated that people act in a boundedly rational way, and their decision making is influenced by various heuristics and biases, either for the good or bad.

Heuristics can aid decision making when people face simple, recurrent problems; they simplify problem-solving by reducing the amount of information to be processed. However, heuristics can also lead to cognitive biases and introduce systematic errors when people face complex decisions.

Research in behavioral economics deals with the effects of psychological, cognitive, emotional, and social factors on judgment and decision making of individuals and organizations, and has repeatedly shown that that the context matters when making judgments or decisions. Thus, people's decisions are not only influenced by the content of the choices, but also the way the choices are presented (i.e., the design of the decision environment), and "nudges"," such as setting defaults or framing decisions can influence people's behavior substantially. Nudging has received increased attention, and in 2017, Richard Thaler has been awarded the Nobel Prize for his pioneering work on nudging.

Today, choices are increasingly made in digital contexts, thus it is important to understand the psychological effects of user-interface designs on people's choices. In particular, it is important to understand how "digital nudging" influences online decision making.

\footnotetext{
${ }^{1}$ Thaler, R.H., \& Sunstein, C.R. (2008). Nudge: Improving Decisions about Health, Wealth, and Happiness. Yale University Press, New Haven \& London.

${ }^{2}$ Weinmann, M., Schneider, C., \& vom Brocke, J. (2016). "Digital Nudging," Business \& Information Systems Engineering, 58(6): pp. 433-436.
}

The main purpose of this minitrack is to explore and extend, as well as exchange, innovative research related to online decision making in the context of information systems design. In particular, this minitrack aims to examine the main applications of behavioral interventions and digital nudges in information-systems design, in particular, research with an emphasis on the effects of interface design on users' behavior, judgment, and decision making in online environments.

\section{This Year's Minitrack}

This year marks the first edition of the minitrack on digital nudging. While the concept of nudging has received much attention in economics, nudging is just beginning to be explored in other disciplines like information systems. Yet as information systems allow to dynamically adapt and personalize content, they provide unique opportunities to implement nudges. Our goal is to provide a platform to discuss cutting-edge research on digital nudging and online decision making.

Given the topic's novelty, research in this area is in its infancy but about to start. This year, we accepted one paper by Ni Huang, Pei-yu Chen, Yili Hong, and Shin-yi Wu. In a field experiment, the researchers found that for nudging messages with a monetary incentive, relational and cognitive capital framings led to increases in social sharing behavior, while nudging messages with simple requests decreased social sharing, compared to the control group without nudging. These findings provide valuable guidelines to effectively nudge social sharing in practice.

Schneider, C., Weinmann, M., \& vom Brocke, J. (2018). "Digital Nudging-Influencing Choices by Using Interface Design," Communications of the ACM, forthcoming. 